\title{
Metastatic breast carcinomas display genomic and transcriptomic heterogeneity
}

Britta Weigelt ${ }^{1,9}$, Charlotte KY Ng${ }^{1,9}$, Ronglai Shen ${ }^{2}$, Tatiana Popova ${ }^{3}$, Michail Schizas ${ }^{4}$, Rachael Natrajan ${ }^{5}$, Odette Mariani ${ }^{3}$, Marc-Henri Stern ${ }^{3,6}$, Larry Norton ${ }^{7}$, Anne Vincent-Salomon ${ }^{3,6}$ and Jorge S Reis-Filho ${ }^{1,8}$

${ }^{1}$ Department of Pathology, Memorial Sloan Kettering Cancer Center, New York, NY, USA; ${ }^{2}$ Department of Epidemiology and Biostatistics, Memorial Sloan Kettering Cancer Center, New York, NY, USA; ${ }^{3}$ INSERM U830, Institut Curie, Paris, France; ${ }^{4}$ Department of Surgery, Memorial Sloan Kettering Cancer Center, New York, NY, USA; ${ }^{5}$ Breakthrough Breast Cancer Research Centre, The Institute of Cancer Research, London, UK; ${ }^{6}$ Institut Curie, Department of Tumor Biology, Paris, France; ${ }^{7}$ Department of Medicine, Memorial Sloan Kettering Cancer Center, New York, NY, USA and ${ }^{8}$ Human Oncology and Pathogenesis Program, Memorial Sloan Kettering Cancer Center, New York, NY, USA

\begin{abstract}
Metaplastic breast carcinoma is a rare and aggressive histologic type of breast cancer, preferentially displaying a triple-negative phenotype. We sought to define the transcriptomic heterogeneity of metaplastic breast cancers on the basis of current gene expression microarray-based classifiers, and to determine whether these tumors display gene copy number profiles consistent with those of BRCA1-associated breast cancers. Twentyeight consecutive triple-negative metaplastic breast carcinomas were reviewed, and the metaplastic component present in each frozen specimen was defined (ie, spindle cell, squamous, chondroid metaplasia). RNA and DNA extracted from frozen sections with tumor cell content $>60 \%$ were subjected to gene expression (IIlumina HumanHT-12 v4) and copy number profiling (Affymetrix SNP 6.0), respectively. Using the best practice PAM50/ claudin-low microarray-based classifier, all metaplastic breast carcinomas with spindle cell metaplasia were of claudin-low subtype, whereas those with squamous or chondroid metaplasia were preferentially of basal-like subtype. Triple-negative breast cancer subtyping using a dedicated website (http://cbc.mc.vanderbilt.edu/tnbc/) revealed that all metaplastic breast carcinomas with chondroid metaplasia were of mesenchymal-like subtype, spindle cell carcinomas preferentially of unstable or mesenchymal stem-like subtype, and those with squamous metaplasia were of multiple subtypes. None of the cases was classified as immunomodulatory or luminal androgen receptor subtype. Integrative clustering, combining gene expression and gene copy number data, revealed that metaplastic breast carcinomas with spindle cell and chondroid metaplasia were preferentially classified as of integrative clusters 4 and 9, respectively, whereas those with squamous metaplasia were classified into six different clusters. Eight of the 26 metaplastic breast cancers subjected to SNP6 analysis were classified as BRCA1-like. The diversity of histologic features of metaplastic breast carcinomas is reflected at the transcriptomic level, and an association between molecular subtypes and histology was observed. BRCA1-like genomic profiles were found only in a subset $(31 \%)$ of metaplastic breast cancers, and were not associated with a specific molecular or histologic subtype.

Modern Pathology (2015) 28, 340-351; doi:10.1038/modpathol.2014.142; published online 21 November 2014
\end{abstract}

Breast cancer is a heterogeneous disease, and distinct molecular approaches for the classification of breast cancers have been described. ${ }^{1-5}$ Triple-

Correspondence: Dr A Vincent-Salomon, MD, PhD, Department of Tumor Biology, Institut Curie, 26 rue d’Ulm, Paris 75248, France or Professor JS Reis-Filho, MD, PhD, FRCPath, Department of Pathology, Memorial Sloan Kettering Cancer Center, 1275 York Avenue, New York, NY 10065, USA.

E-mail: anne.salomon@curie.fr or reisfilj@mskcc.org

${ }^{9}$ These authors contributed equally to this work.

Received 8 June 2014; accepted 14 August 2014; published online 21 November 2014 negative breast cancers (ie, tumors that lack expression of estrogen receptor (ER), progesterone receptor (PR), and HER2) have been shown to comprise a heterogeneous collection of tumors at the morphologic and molecular levels. ${ }^{6-9}$ Not only can triple-negative breast cancers be classified into the 'intrinsic' molecular subtypes (ie, luminal A, luminal B, basal-like, HER2-enriched, normal breast-like, and claudin-low), ${ }^{1,2}$ but also six transcriptomic subtypes of triple-negative breast cancer with potential therapeutic implications (ie, basallike 1, basal-like 2, immunomodulatory, luminal 
androgen receptor, mesenchymal-like, and mesenchymal stem-like) ${ }^{3,10}$ have been described. In addition, triple-negative breast cancers are represented in most of the 10 'integrative' subgroups obtained through integrative analysis of copy number and gene expression data, ${ }^{5}$ and a subset of these tumors have been shown to be classified as BRCA1-like on the basis of SNP array-based methods. ${ }^{4}$

Metaplastic breast carcinoma is a rare and aggressive histologic type of breast cancer, preferentially of triple-negative phenotype. ${ }^{11-13}$ The term metaplastic breast carcinoma is descriptive, as it encompasses a heterogeneous group of tumors characterized by the presence of malignant cells, showing differentiation towards squamous epithelium, or mesenchymal elements, such as spindle, chondroid, osseous and rhabdoid differentiation. ${ }^{12,13}$ Metaplastic breast cancers display a rather aggressive clinical behavior, and, unlike other forms of triple-negative breast cancers, these tumors seem not to respond to conventional chemotherapy regimens. ${ }^{14}$ Interestingly, our group ${ }^{15,16}$ and others $^{17}$ have demonstrated that tumors arising in the mouse mammary gland of conditional mouse models, where inactivation of the tumor suppressor genes Brca1 and Trp53 was driven by $\beta$ lactoglobulin or cytokeratin 14 (Krt14), have histologic features that closely recapitulate those of human metaplastic breast carcinomas, suggesting that loss of BRCA1 function or loss of homologous recombination DNA repair of double-strand breaks may have a role in the development of metaplastic breast carcinomas.

The molecular classification of metaplastic breast carcinomas has been proven controversial. Whereas our group previously observed that these tumors largely resembled basal-like breast cancer at the transcriptomic level, ${ }^{18,19}$ others have suggested that the vast majority of these cancers would either constitute a distinct subtype ${ }^{20}$ or be classified as of claudin-low subtype. ${ }^{2}$ We posited that different subtypes of metaplastic breast cancers would differ in their transcriptomic profiles according to their differentiation patterns; therefore, one could hypothesize that the differences observed in the molecular classification of metaplastic breast carcinomas in different studies would stem from differences in the type of tumors analyzed (eg, in Hennessy et $a l,{ }^{20}$ all tumors had sarcomatoid or squamous metaplasia). To address this hypothesis, we subjected 28 metaplastic breast carcinomas to microarray-based gene expression profiling and SNP analyses to define the molecular subtypes of these cancers using current microarraybased classifiers. Furthermore, given the histologic features of mammary gland tumors in conditional mouse models of Brca1 inactivation, we sought to investigate whether human metaplastic breast carcinomas display gene copy number profiles consistent with those of BRCA1-associated breast cancers.

\section{Materials and methods}

\section{Tissue Samples}

Twenty-eight consecutive triple-negative metaplastic breast carcinomas were retrieved from the tumor banks of the authors' institutions. The diagnostic slides were reviewed by two pathologists who were members of the latest World Health Organization panel for the classification of tumors of the breast (AV-S, a diagnostic surgical pathologist with an interest in breast pathology, and JSR-F, an academic experimental pathologist with an interest in breast pathology), and diagnosed according to the latest World Health Organization classification ${ }^{12}$ into three groups: metaplastic breast carcinomas with squamous metaplasia, with mesenchymal elements, and spindle cell carcinomas. In addition, representative sections of the frozen material of each metaplastic breast cancer were reviewed and the tumor cell content and composition of the metaplastic elements were estimated (ie, spindle cell metaplasia, squamous metaplasia and chondroid metaplasia). In each frozen sample, the metaplastic component most abundantly present was defined (Table 1). This study was approved by the local institutional review boards of the authors' institutions. Tumors were graded according to the Nottingham grading system. ${ }^{21}$ RNA and DNA were extracted from representative frozen sections of each tumor, all of which contained $>60 \%$ of cancer cells as defined by histologic analysis, using Trizol (Invitrogen) and the DNeasy Blood and Tissue Kit (Qiagen), respectively, as previously described. ${ }^{22-24}$ RNA and DNA of sufficient quality and quantity for downstream analyses were obtained from 28 and 26 cases, respectively (Supplementary Table S1). Tumor cellularity estimated by histologic analysis was further assessed based on the gene copy number data (SNP 6.0, see below) using the ABSOLUTE algorithm. ${ }^{25}$

\section{Immunohistochemistry}

Immunohistochemical profiles of the 28 metaplastic breast carcinomas were assessed on 3- $\mu$ m-thick sections, using antibodies against ER, PR, and HER2, as previously described. ${ }^{24}$ Positive and negative controls were included in each experiment. Immunohistochemical analyses were interpreted by two pathologists (AV-S and JSR-F) according to the ASCO/CAP guidelines. ${ }^{26,27}$

\section{Gene Expression and Gene Copy Number Profiling and Analysis}

Gene expression profiling and gene copy number analysis were performed using the HumanHT-12 v4 platform (Illumina) and the Human SNP 6.0 arrays (Affymetrix), respectively, as previously described. ${ }^{4,28}$ Publicly available gene expression data from 997 
Table 1 Morphologic types of metaplastic breast carcinomas analyzed in this study

\begin{tabular}{|c|c|c|c|c|c|c|c|c|c|}
\hline \multirow[b]{2}{*}{ Sample ID } & \multirow[b]{2}{*}{ Diagnosis } & \multirow[b]{2}{*}{$\begin{array}{l}\text { Histologic } \\
\text { grade }^{\mathrm{a}}\end{array}$} & \multirow[b]{2}{*}{$\begin{array}{l}\text { Triple-negative } \\
\text { phenotype }^{\mathrm{b}}\end{array}$} & \multicolumn{4}{|c|}{ Metaplastic element present (diagnostic) } & \multirow[b]{2}{*}{$\begin{array}{l}\text { Matrix- } \\
\text { producing }\end{array}$} & \multirow[b]{2}{*}{$\begin{array}{l}\text { Component in } \\
\text { frozen sample }\end{array}$} \\
\hline & & & & Spindle & Squamous & Chondroid & Osseous & & \\
\hline META33 & $\begin{array}{l}\text { Carcinoma with } \\
\text { mesenchymal elements }\end{array}$ & 3 & Yes & & & + & & & Chondroid \\
\hline META52 & $\begin{array}{l}\text { Carcinoma with } \\
\text { mesenchymal elements }\end{array}$ & 3 & Yes & + & & + & & & Chondroid \\
\hline META32 & $\begin{array}{l}\text { Carcinoma with } \\
\text { mesenchymal elements }\end{array}$ & 3 & Yes & + & & & + & & Spindle \\
\hline META39 & $\begin{array}{l}\text { Carcinoma with } \\
\text { mesenchymal elements }\end{array}$ & 3 & Yes & + & & + & & & Spindle \\
\hline META58 & $\begin{array}{l}\text { Carcinoma with } \\
\text { mesenchymal elements }\end{array}$ & 3 & Yes & + & & & + & & Spindle \\
\hline META59 & $\begin{array}{l}\text { Carcinoma with } \\
\text { mesenchymal elements }\end{array}$ & 3 & Yes & + & & + & + & & Spindle \\
\hline META41 & $\begin{array}{l}\text { Carcinoma with } \\
\text { mesenchymal elements }\end{array}$ & 3 & Yes & & + & + & & & Squamous \\
\hline META43 & $\begin{array}{l}\text { Carcinoma with } \\
\text { mesenchymal elements }\end{array}$ & 3 & Yes & + & + & + & & & Squamous \\
\hline META62 & $\begin{array}{l}\text { Carcinoma with } \\
\text { mesenchymal elements }\end{array}$ & 3 & Yes & + & + & + & & & Squamous \\
\hline META30 & $\begin{array}{l}\text { Carcinoma with } \\
\text { mesenchymal elements }\end{array}$ & 3 & Yes & & & + & & + & Chondroid \\
\hline META31 & $\begin{array}{l}\text { Carcinoma with } \\
\text { mesenchymal elements }\end{array}$ & 2 & Yes & & & + & & + & Chondroid \\
\hline META53 & $\begin{array}{l}\text { Carcinoma with } \\
\text { mesenchymal elements }\end{array}$ & 3 & Yes & & & + & & + & Chondroid \\
\hline META64 & $\begin{array}{l}\text { Carcinoma with } \\
\text { mesenchymal elements }\end{array}$ & 3 & Yes & & & + & & + & Chondroid \\
\hline META36 & $\begin{array}{l}\text { Carcinoma with } \\
\text { mesenchymal elements }\end{array}$ & 2 & Yes & & & + & & + & Chondroid \\
\hline META55 & $\begin{array}{l}\text { Carcinoma with } \\
\text { mesenchymal elements }\end{array}$ & 3 & Yes & & & + & & + & Chondroid \\
\hline META44 & $\begin{array}{l}\text { Carcinoma with } \\
\text { mesenchymal elements }\end{array}$ & 3 & Yes & & + & + & & + & Squamous \\
\hline META42 & $\begin{array}{l}\text { Carcinoma with squamous } \\
\text { metaplasia }\end{array}$ & 3 & Yes & & + & & & & Squamous \\
\hline META46 & $\begin{array}{l}\text { Carcinoma with squamous } \\
\text { metaplasia }\end{array}$ & 3 & Yes & & + & & & & Squamous \\
\hline META34 & $\begin{array}{l}\text { Carcinoma with squamous } \\
\text { metaplasia }\end{array}$ & 3 & Yes & & + & & & & Squamous \\
\hline META37 & $\begin{array}{l}\text { Carcinoma with squamous } \\
\text { metaplasia }\end{array}$ & 3 & Yes & + & + & & & & Squamous \\
\hline META47 & Spindle cell carcinoma & 3 & Yes & + & & & & & Spindle \\
\hline META49 & Spindle cell carcinoma & 3 & Yes & + & & & & & Spindle \\
\hline META56 & Spindle cell carcinoma & 3 & Yes & + & & & & & Spindle \\
\hline META57 & Spindle cell carcinoma & 3 & Yes & + & & & & & Spindle \\
\hline META60 & Spindle cell carcinoma & 3 & Yes & + & & & & & Spindle \\
\hline META65 & Spindle cell carcinoma & 3 & Yes & + & & & & & Spindle \\
\hline META45 & Spindle cell carcinoma & 3 & Yes & + & + & & & & Squamous \\
\hline META40 & Spindle cell carcinoma & 3 & Yes & + & + & & & & Squamous \\
\hline
\end{tabular}

+ , present.

${ }^{a}$ Histologic grading was performed according to Nottingham grading index. ${ }^{21}$

bTriple-negative phenotype: lack of estrogen receptor, progesterone receptor, and HER2 expression.

invasive ductal carcinomas of no special type were retrieved from the METABRIC discovery cohort, ${ }^{5}$ of which 134 were classified as of triple-negative phenotype based on the gene expression of ESR1, $P g R$, and HER2, as previously described. ${ }^{3}$ Gene expression data of the 28 metaplastic breast carcinomas and 997 METABRIC invasive ductal carcinomas of no special type were normalized as described by Curtis et al $1^{5}$ (see Supplementary R code and script). In brief, the 'IlluminaID' was used to match probes from the HumanHT-12 v4 and HumanHT-12 v3 platforms. A target distribution was generated for normalization to avoid the influence of sub-optimal probes. Probes were selected on the basis that they were a perfect genomic match with a GC content between 38 and $64 \%$, according to the re-annotation of the Illumina HT-12 v3 platform, ${ }^{29}$ did not target sex chromosomes or genes in the PAM50 gene list, did not contain SNPs or a polyG tail, and did not have multiple genomic matches. ${ }^{5}$ ER-positive and ER-negative samples were quantile normalized separately and averaged to obtain the final target distribution. The values for the remaining probes were obtained by interpolation using the weighted-normalized 
intensities of the target distribution probes with most similar intensities prior to normalization.

After normalization using Genotyping Console (Affymetrix), SNP6 arrays were processed using the Genome Alteration Print method to obtain absolute copy number profiles. ${ }^{30}$ Tumor ploidy was set to 2 (DNA index $<1.3$ ) or 4 (DNA index $\geq 1.3$ ) as previously described. ${ }^{4}$ The tumor cell content was estimated on the basis of the gene copy number and allele B frequency using the ABSOLUTE algorithm as previously described. ${ }^{25}$

The raw and processed gene expression data as well as the gene copy number data are available on Gene Expression Omnibus (reference GSE 57549), and the $\mathrm{R}$ script and code are available in the Supplementary Materials.

\section{Intrinsic Molecular Subtyping}

PAM50 intrinsic molecular subtyping was performed using the scripts accompanying the METABRIC study by Curtis et al. ${ }^{5}$ In brief, 100 random reference distributions consisting of all ER-negative samples and equal number of randomly selected ERpositive samples were defined during the median centering step. At each iteration, the Spearman's rank correlation between each sample and each PAM50 centroid retrieved from the METABRIC study was assigned. The consensus of the 100 iterations determined the final PAM50 molecular subtype of a given case. ${ }^{5}$ Claudin-low subtyping was performed using a modified version of the PAM50 classification scripts from the METABRIC study to include the claudin-low centroid. For the claudin-low subtyping, Euclidean distance was used according to the University of North Carolina (UNC) guidelines (http://genome.unc.edu/pubsup/ breastGEO/Guide\% 20to\%20Intrinsic\% 20Subtyping\% 209-6-10.pdf). Intrinsic molecular subtypes were determined by integrating PAM50 and claudin-low subtyping by strictly following the UNC guidelines. The PAM50 intrinsic subtyping method used here showed an almost perfect agreement (Rand index $=0.955$, unweighted Kappa $=0.941$ ), with the classification published by Curtis et $a l^{5}$ (Supplementary Table S2).

\section{Triple-negative Breast Cancer Subtyping}

Triple-negative breast cancer subtyping was performed on the combined data sets of metaplastic breast carcinomas and the triple-negative invasive ductal carcinomas of no special type from the METABRIC discovery cohort using a dedicated webserver (http://cbc.mc.vanderbilt.edu/tnbc/). ${ }^{3}$ Given that this algorithm requires $>75 \%$ of the probes' expression to be lower than those of the ESR1 probe(s), 1 of the 28 metaplastic breast carcinomas and 8 of the 134 triple-negative invasive ductal carcinomas of no special type were not classifiable using this approach.

\section{Integrative Cluster Analysis}

To define the integrative subtype of each metaplastic breast carcinoma included in this study, we used the set of 754 features (39 gene copy number aberrations and 715 gene expression values) originally derived in the discovery cohort reported by Curtis et $a l^{5}$ and used to classify the validation set. After standardizing the gene expression features in the metaplastic breast cancer data set, the Pearson's correlation distance between the profiles of each metaplastic breast carcinoma (gene copy number and gene expression) and each of the 10 centroids for the integrative subtypes described by Curtis et al ${ }^{5}$ was computed. The centroid with the smallest correlation distance to each sample was defined as its integrative subtype. The same methodology was used to define the integrative subtype of each of the 134 triple-negative invasive ductal carcinomas of no special type from the METABRIC discovery cohort. ${ }^{5}$ The integrative subtyping method used here for the classification of the metaplastic breast carcinomas and triple-negative invasive ductal carcinomas of no special type and the method used by Curtis et $a l^{5}$ showed very good agreement (Rand index $=0.93$, unweighted Kappa $=0.804$; Supplementary Table S2).

\section{BRCAness Classification}

BRCAness classification was performed based on the number of Large-scale State Transitions in tumor genomic profiles as previously described. ${ }^{4}$ In brief, a large-scale state transition was defined as a chromosomal break (ie, change in copy number of major allele counts) between adjacent regions of at least $10 \mathrm{Mb}$ obtained after smoothing and filtering small-scale copy number variations $(<3 \mathrm{Mb})$. Tumor profiles were assigned to genomic BRCAness or 'BRCA1-like' if the number of large-scale state transitions were $\geq 15$ (ploidy 2, near-diploid tumors) or 20 (ploidy 4, near-tetraploid tumors). BRCAness classification was performed for the 26 metaplastic breast carcinomas for which copy number profiling was performed, as well as for the 134 triple-negative invasive ductal carcinomas of no special type from the METABRIC study.

\section{Results}

\section{Molecular Subtyping Reveals Heterogeneity of Metaplastic Breast Carcinomas}

Of the 28 metaplastic breast carcinomas included in this study, 16 were diagnosed as metaplastic breast carcinomas with mesenchymal elements, 7 of which were matrix-producing, 4 as metaplastic breast 


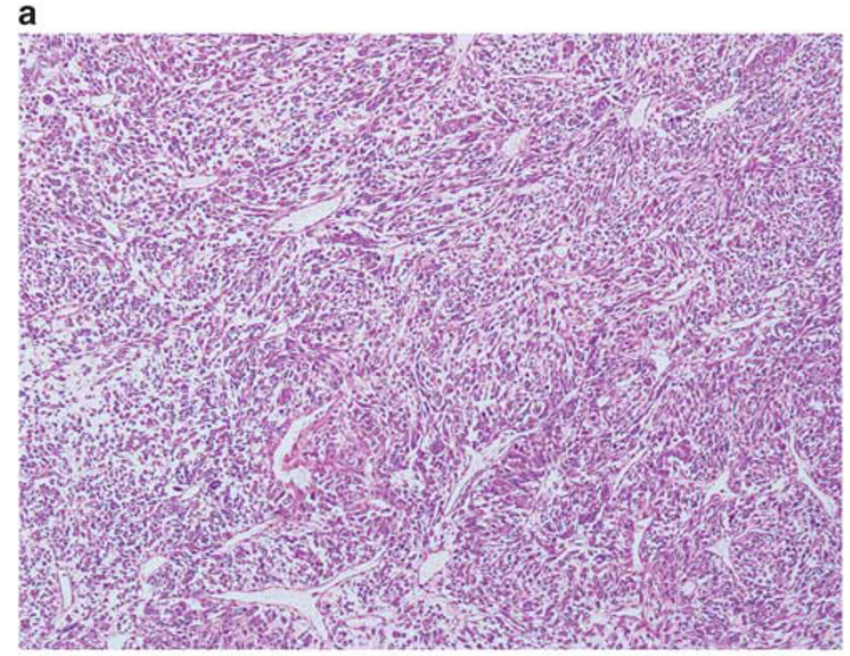

b

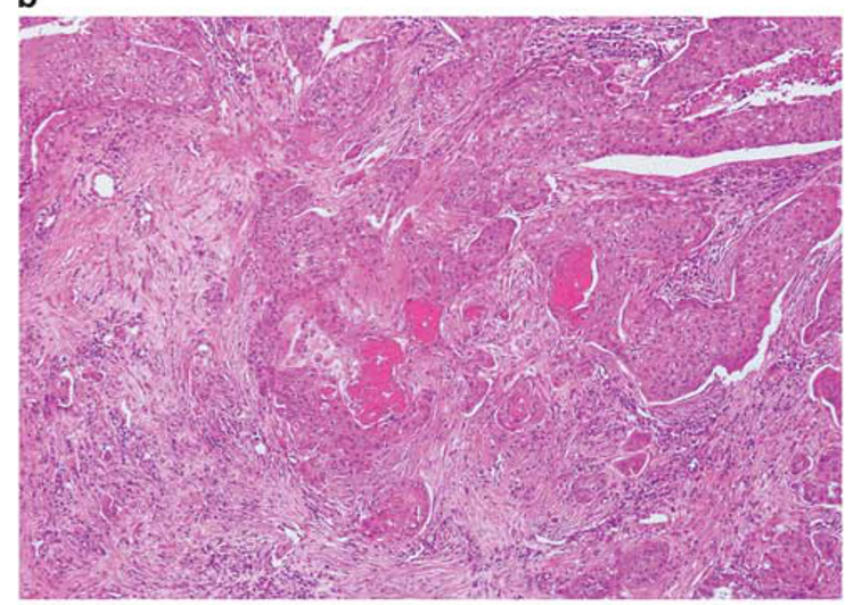

c

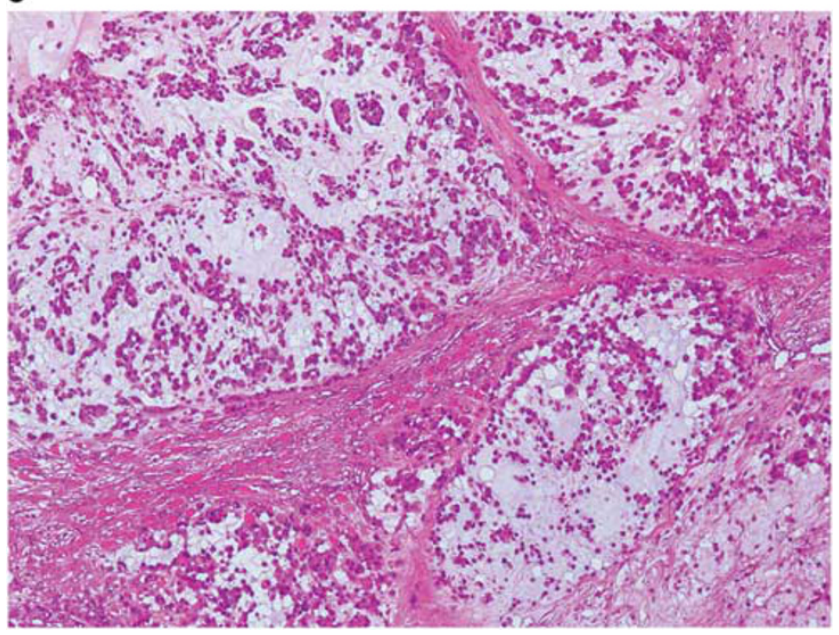

Figure 1 Representative micrographs of histologic subtypes of metaplastic breast cancers included in this study. (a) Metaplastic breast carcinoma with spindle cell metaplasia, (b) with squamous metaplasia, and (c) with mesenchymal elements (chondroid metaplasia). Original magnification $\times 40$.

carcinomas with squamous metaplasia and 8 as spindle cell carcinomas (Figure 1, Table 1). Metaplastic breast cancer is generally considered to be of high grade, ${ }^{31}$ and $93 \%$ and $7 \%$ of the cases analyzed here were of Nottingham histologic grades 3 and 2, respectively (Table 1). No case was classified as fibromatosis-like metaplastic breast carcinoma. ${ }^{32,33}$

'Intrinsic' molecular subtyping revealed that the majority of metaplastic breast carcinomas (15/28; $54 \%)$ were of claudin-low subtype, and $36 \%(10 / 28)$ and $11 \%(3 / 28)$ of basal-like and normal breast-like subtypes, respectively (Table 2, Supplementary Table S1). When metaplastic breast carcinomas were classified into the triple-negative breast cancer subtypes, we observed that $43 \%(12 / 28)$ and $14 \%$ $(4 / 28)$ were of mesenchymal-like and mesenchymal stem-like subtypes, respectively. Only a subset of metaplastic breast carcinomas was of basal-like 1 $(1 / 28 ; 4 \%)$ or basal-like $2(2 / 28 ; 7 \%)$ triple-negative breast cancer subtypes, and $29 \%(8 / 28)$ were classified as unstable. It should be noted that in contrast to 'common type' triple-negative breast cancers (ie, grade 3 invasive ductal carcinoma of no special type), ${ }^{3,10}$ none of the metaplastic breast carcinomas analyzed here were of immunomodulatory or luminal androgen receptor triple-negative breast cancer subtypes (Table 2; see below). Furthermore, only a subset of metaplastic breast carcinomas $(8 / 26 ; 31 \%)$ displayed a BRCAness signature, whereas the majority of cases $(13 / 26 ; 50 \%)$ did not harbor a pattern of large-scale state transitions consistent with that found in BRCA1/2 breast cancers, or were unclassifiable $(5 / 26 ; 19 \%)$.

The molecular heterogeneity observed in the classification systems on the basis of gene expression profiling was further corroborated when the 26 metaplastic breast carcinomas, for which both gene expression and gene copy number data were available, were subjected to integrative cluster analysis. ${ }^{5}$ This analysis revealed that $2(8 \%), 8(31 \%)$, and 3 $(12 \%)$ metaplastic breast cancers were of integrative cluster (IntClust) 3, IntClust 4, and IntClust 8, respectively, which have been associated with low/ intermediate genomic instability and low histologic grade, ${ }^{5,34}$ whereas $4(15 \%), 1(4 \%), 4(15 \%)$, and 4 (15\%) metaplastic breast cancers were of IntClust 1, IntClust 5, IntClust 9, and IntClust 10, respectively, which have been shown to be associated with intermediate/high genomic instability and high histologic grade ${ }^{5,34}$ (Table 2, Supplementary Table S1). Consistent with these observations, the two histologic grade 2 metaplastic breast carcinomas described in this study were classified as of IntClust 3 and 8 and both were classified as not harboring a BRCAness genomic signature.

Basal-like and claudin-low metaplastic breast carcinomas were found to display differences in regard to their classification into the six triple-negative breast cancer subtypes, integrative subtypes, and BRCAness signature. Whereas metaplastic breast carcinomas of basal-like intrinsic molecular subtype were significantly more frequently classified as of mesenchymal-like triplenegative breast cancer subtype (7/10; 70\%; Fisher's 
Table 2 Molecular subtypes of metaplastic breast carcinomas

\begin{tabular}{|c|c|c|c|c|}
\hline & \multirow{2}{*}{$\begin{array}{l}\text { All metaplastic breast } \\
\text { carcinomas }(\mathrm{n}=28)\end{array}$} & \multicolumn{3}{|c|}{ Metaplastic component in frozen specimen } \\
\hline & & Chondroid $(\mathrm{n}=8)$ & Spindle $(\mathrm{n}=10)$ & Squamous $(\mathrm{n}=10)$ \\
\hline \multicolumn{5}{|c|}{ PAM50/ Claudin-low $(\mathrm{n}=28)$} \\
\hline Basal-like & 10 & 5 & & 5 \\
\hline Normal breast-like & 3 & 2 & & 1 \\
\hline Claudin-low & 15 & 1 & 10 & 4 \\
\hline \multicolumn{5}{|c|}{ Triple-negative breast cancer subtypes $(\mathrm{n}=28)$} \\
\hline Mesenchymal-like & 12 & 8 & 1 & 3 \\
\hline Basal-like 1 & 1 & & & 1 \\
\hline Basal-like 2 & 2 & & & 2 \\
\hline \multicolumn{5}{|l|}{ Immunomodulatory } \\
\hline Mesenchymal stem-like & 4 & & 4 & \\
\hline \multicolumn{5}{|c|}{ Luminal androgen receptor } \\
\hline Unstable & 8 & & 5 & 3 \\
\hline Not classifiable & 1 & & & 1 \\
\hline \multicolumn{5}{|c|}{ Integrative clustering $(\mathrm{n}=26)$} \\
\hline Integrative cluster 1 & 4 & & 3 & 1 \\
\hline \multicolumn{5}{|l|}{ Integrative cluster 2} \\
\hline Integrative cluster 3 & 2 & 1 & & 1 \\
\hline Integrative cluster 4 & 8 & & 6 & 2 \\
\hline Integrative cluster 5 & 1 & & & 1 \\
\hline \multicolumn{5}{|l|}{ Integrative cluster 6} \\
\hline \multicolumn{5}{|l|}{ Integrative cluster 7} \\
\hline Integrative cluster 8 & 3 & 2 & & 1 \\
\hline Integrative cluster 9 & 4 & 4 & & \\
\hline Integrative cluster 10 & 4 & 1 & 1 & 2 \\
\hline \multicolumn{5}{|l|}{ BRCAness $(\mathrm{n}=26)$} \\
\hline BRCAness & 8 & 2 & 1 & 5 \\
\hline Non-BRCAness & 13 & 6 & 4 & 3 \\
\hline Unclassified & 5 & & 5 & \\
\hline
\end{tabular}

Gene expression and gene copy number data were available for 28 and 26 metaplastic breast carcinomas, respectively.

exact test, $P=0.0344$ ), metaplastic breast carcinomas of claudin-low intrinsic subtype were more heterogeneous and only 3 out of 15 cases (20\%) were classified as of mesenchymal-like subtype, with the remaining cases being of basal-like $2(2 / 15 ; 13 \%)$, mesenchymal stem-like $(4 / 15 ; 27 \%)$, or unstable $(6 /$ $15 ; 40 \%$ ) triple-negative breast cancer subtype (Figure 2, Supplementary Table S3). Interestingly, all triple-negative breast cancer subtypes could be found in the group of IntClust 4 metaplastic breast carcinomas, with the exception of the basal-like 1 type, whereas IntClust 9 and IntClust 10 metaplastic breast carcinomas were of mesenchymal-like triplenegative breast cancer subtype (Figure 2, Supplementary Table S3).

One of the confounding variables in the use of genomic classifiers is the percentage of tumor cells within each sample. ${ }^{35}$ To rule out this potential confounding variable, we ascertained the tumor cell content not only with semi-quantitative histologic assessment, but also using ABSOLUTE and investigated whether there would be differences between tumors classified as basal-like, claudin-low and normal breast-like, according to the triple-negative breast cancer subtypes, and between IntClust 4 and other integrative cluster subtypes. These analyses revealed no statistically significant differences in tumor cell content according to specific subtypes (Kruskal-Wallis, $P>0.1$; Supplementary Figure S1).

Taken together, molecular subtyping of metaplastic breast carcinomas using current microarraybased classifiers demonstrates that these tumors are not only heterogeneous at the morphologic but also at the molecular level.

\section{Metaplastic Breast Carcinomas with Distinct Metaplastic Elements Display Different Molecular Subtypes}

We next sought to define whether the different morphologic types of metaplastic breast carcinoma would be more homogeneous at the genomic and transcriptomic levels than metaplastic breast carcinomas as a group. Given that upon histologic review of the frozen samples available, each sample was preferentially composed of one metaplastic component only, and that previous analyses carried out by our group revealed that genomic differences may be observed between morphologically distinct components of metaplastic breast cancers, ${ }^{24}$ we focused our comparisons on the basis of the histologic 


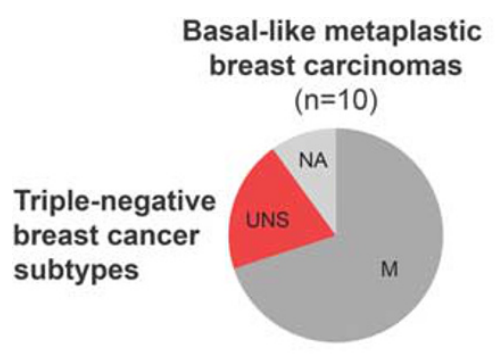

\section{Claudin-low metaplastic}

breast carcinomas
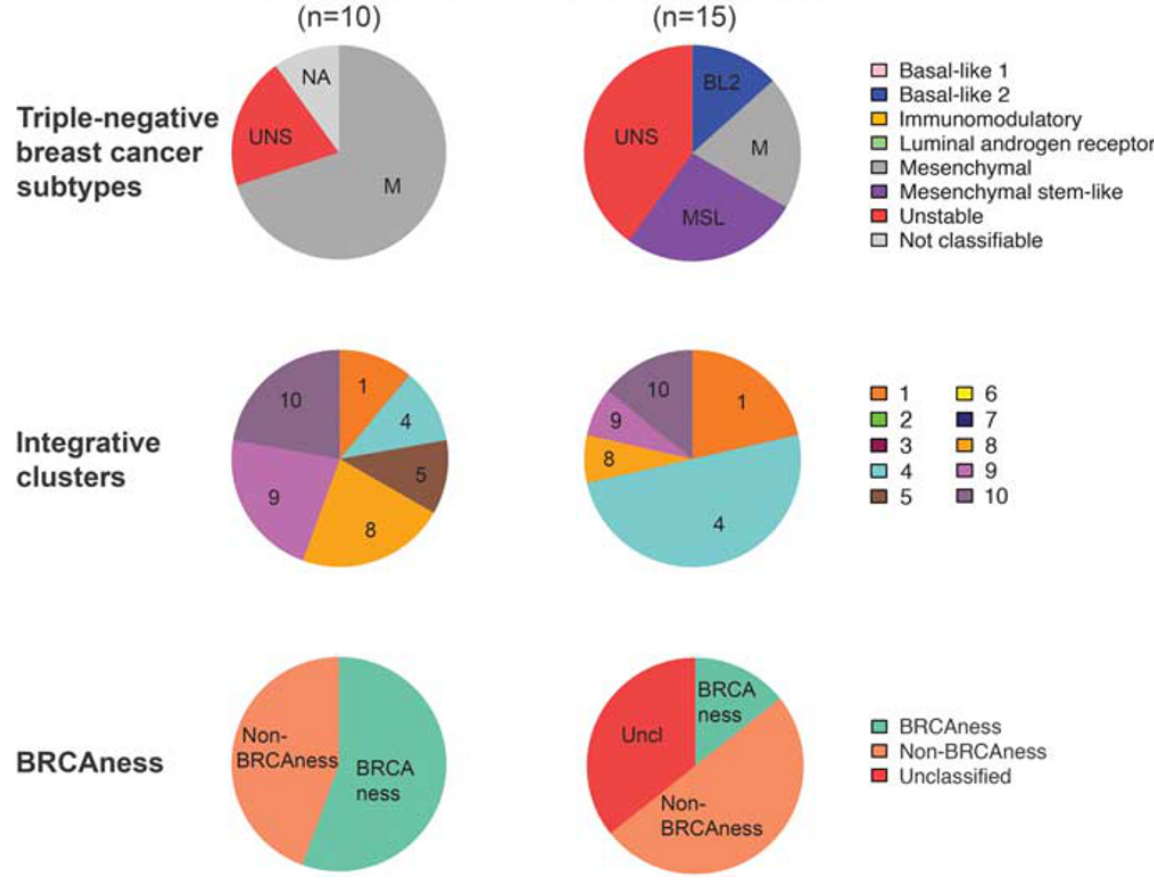

Figure 2 Metaplastic breast cancers of basal-like and claudin-low intrinsic subtypes are distinct at the molecular level. Only metaplastic breast cancers classified into basal-like or claudin-low intrinsic molecular subtypes were included in this analysis. Pie charts representing the triple-negative breast cancer subtypes, the integrative cluster and the BRCAness composition of these metaplastic breast carcinomas are shown. For integrative cluster and BRCAness analysis, only cases with available SNP6 data were included (ie, 9 basal-like and 14 claudin-low metaplastic breast carcinomas), given that SNP6 data are required for subtyping using these classification systems.

component present in the frozen tissue specimen. Cases were then classified according to the component present in the frozen sample (chondroid $n=8$, spindle $n=10$, and squamous $n=10$, Table 1 ).

This subgroup analysis revealed that metaplastic breast carcinomas with spindle cell metaplasia (ie, metaplastic carcinoma where the frozen sample analyzed was predominantly or completely composed of metaplastic spindle cells) were homogeneous in regard to the intrinsic molecular subtypes, given that all cases were of claudin-low subtype (Fisher's exact test, $P<0.001$, Figure 3, Table 2). By contrast, metaplastic breast carcinomas with squamous and chrondroid metaplasia were more heterogeneous and were classified as basal-like, normal breast-like or claudin-low subtypes. Furthermore, as compared with other types of metaplastic breast carcinomas, metaplastic breast carcinomas with spindle cell metaplasia were significantly more frequently of mesenchymal stem-like $(40 \%)$ or unstable $(50 \%)$ triple-negative breast cancer subtypes (Fisher's exact test, $P=0.001$ ), and of IntClust 4 (64\%; Fisher's exact test, $P=0.021$ ) associated with low genomic instability and low histologic grade $^{5,34}$ (Figure 3, Supplementary Table S1). Metaplastic breast carcinomas with chrondroid metaplasia were homogeneous in regard to the six triplenegative breast cancer subtype classification, given that all samples were classified as of mesenchymal- like subtype (Fisher's exact test, $P<0.01$ ), but heterogeneous in regard to the integrative clustering subtypes (IntClusts 3, 8, 9, and 10), and 75\% of cases displayed a non-BRCAness signature (Figure 3). Metaplastic breast carcinomas with squamous metaplasia were rather heterogeneous at the transcriptomic and genomic levels as revealed by the molecular classifiers assessed here. These tumors were of basal-like $(50 \%)$, normal breast-like $(10 \%)$, or claudin-low (40\%) intrinsic molecular subtypes, and triple-negative breast cancer subtyping revealed that metaplastic breast carcinomas with squamous metaplasia had mesenchymal-like (30\%), basal-like $1(10 \%)$, basal-like $2(20 \%)$, or unstable $(30 \%)$ triplenegative breast cancer phenotypes. Furthermore, metaplastic breast carcinomas with squamous metaplasia were of IntClusts 1, 3, 4, 5, 8, or 10, and the majority $(5 / 8 ; 62 \%)$ displayed a BRCAness signature (Figure 3).

It should be noted, however, that the associations unraveled between the metaplastic elements of the metaplastic breast carcinomas and the distinct molecular classifications tested here are less clear, if the diagnosis based upon review of the diagnostic formalin-fixed paraffin-embedded slides rather than the metaplastic component present in the frozen specimen would be used (Supplementary Figure S2). In fact, metaplastic breast carcinomas with mesenchymal elements, with squamous differ- 


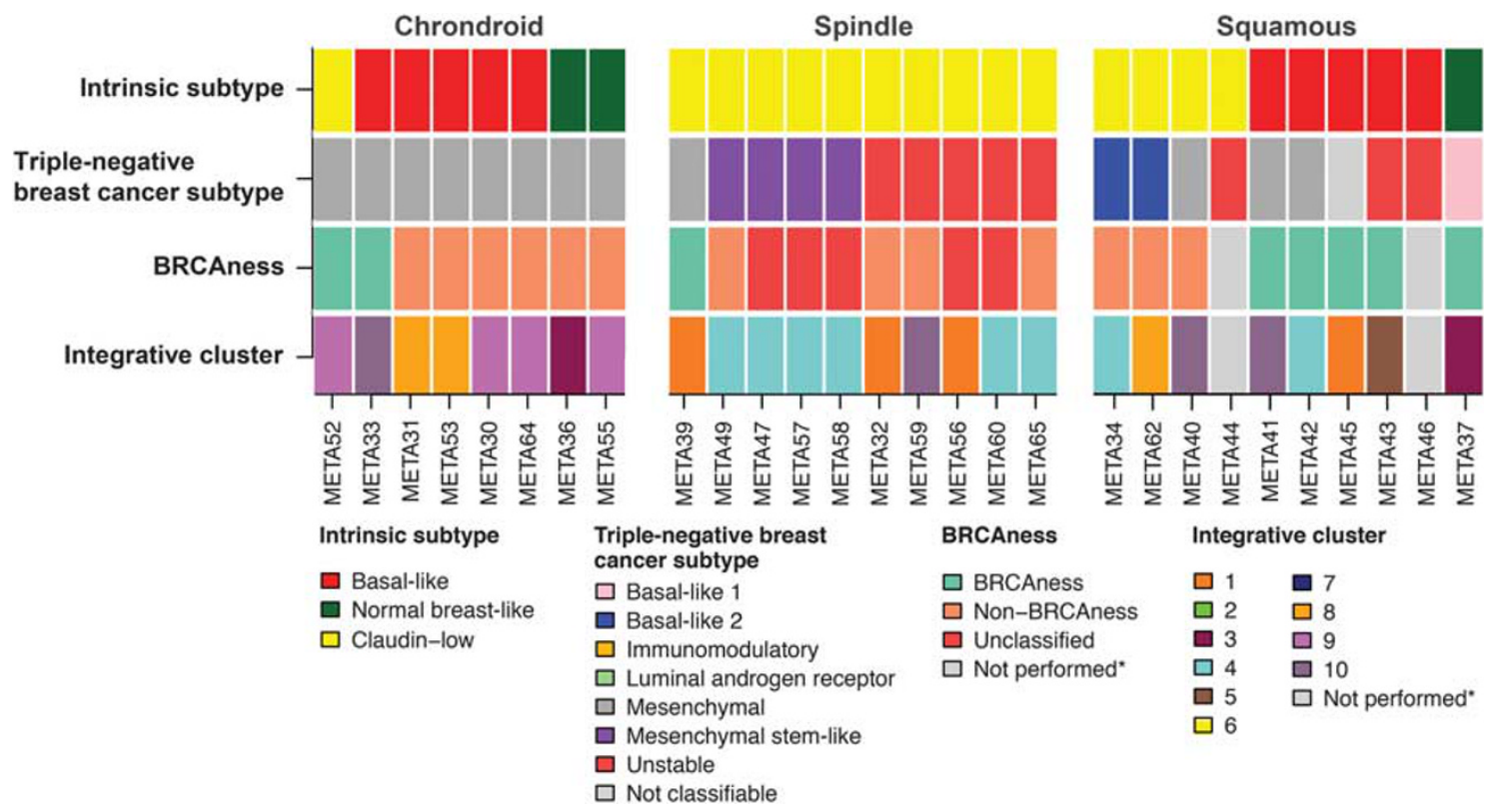

Figure 3 Metaplastic elements display distinct molecular subtypes. Classification of the metaplastic components present in the frozen sample from each metaplastic breast cancer subjected to molecular profiling in this study. The PAM50 intrinsic molecular subtype, triplenegative breast cancer subtype, BRCAness signature and integrative cluster for each case are shown. *: No SNP6 data available.

entiation, and spindle cell carcinomas were all heterogeneous in regard to the different molecular classifiers assessed, with the exception of metaplastic spindle cell carcinomas, which, in a way akin to the analysis of spindle cell metaplasia from the frozen samples (described above), were preferentially of claudin-low intrinsic molecular subtype (8/ 9, 89\%; Fisher's exact test, $P=0.04$; Supplementary Figure S2).

Taken together, our data demonstrate that the histologic type of metaplastic elements in metaplastic breast carcinomas may determine the results of genomic and transcriptomic classifiers, and that the molecular subtype of a given metaplastic breast carcinoma may be determined by the metaplastic component subjected to molecular analysis. Samples of metaplastic breast carcinomas preferentially composed of spindle cell and chrondroid metaplasia were homogeneous and exclusively of claudin-low 'intrinsic' molecular subtype and mesenchymal-like triple-negative breast carcinoma subtype, respectively.

\section{Molecular Subtypes of Metaplastic Breast Carcinomas are Distinct from those of Invasive Ductal Carcinomas of no Special Type of Triple-Negative Phenotype}

We sought to define whether the molecular subtypes of metaplastic breast carcinomas would be distinct from those of triple-negative invasive ductal carcinomas of no special type. Comparative analysis of the metaplastic breast carcinomas included in this study and the 134 triple-negative invasive ductal carcinomas of no special type from the METABRIC discovery cohort ${ }^{5}$ revealed significant differences. In contrast to triple-negative metaplastic breast carcinomas, which are preferentially of claudinlow (54\%) and basal-like (36\%) intrinsic subtypes, triple-negative invasive ductal carcinomas of no special type are preferentially of basal-like subtype (69\%), but also of HER2-enriched (17\%), claudinlow $(7 \%)$, normal breast-like $(5 \%)$, and luminal A (2\%) subtypes (10/28 basal-like metaplastic breast carcinomas vs 92/134 basal-like triple-negative invasive ductal carcinomas of no special type, Fisher's exact test, $P=0.0021 ; 15 / 28$ claudin-low metaplastic breast carcinomas vs 9/134 claudin-low triple-negative invasive ductal carcinomas of no special type, Fisher's exact test, $P<0.001$; Figure 4). Likewise, whereas the majority of metaplastic breast carcinomas were of mesenchymal-like (43\%), mesenchymal stem-like (14\%), and unstable (29\%) triple-negative breast cancer subtypes, and none of immunomodulatory and luminal androgen receptor subtypes, triple-negative invasive ductal carcinomas of no special type were found to be more heterogeneous and to display all triple-negative breast cancer subtypes, with the immunomodulatory (36/ 126, 29\%), basal-like $1(30 / 126,24 \%)$, luminal androgen receptor $(20 / 126,16 \%)$, and mesenchymal-like $(15 / 126,12 \%)$ subtypes being the most frequent (Figure 4). Interestingly, when triple-negative invasive ductal carcinomas of no special type were subjected to integrative clustering analysis, the majority of cases (89/134, 66\%) were of IntClust 10, which is associated with high histologic grade and intermediate genomic instability, whereas only a 


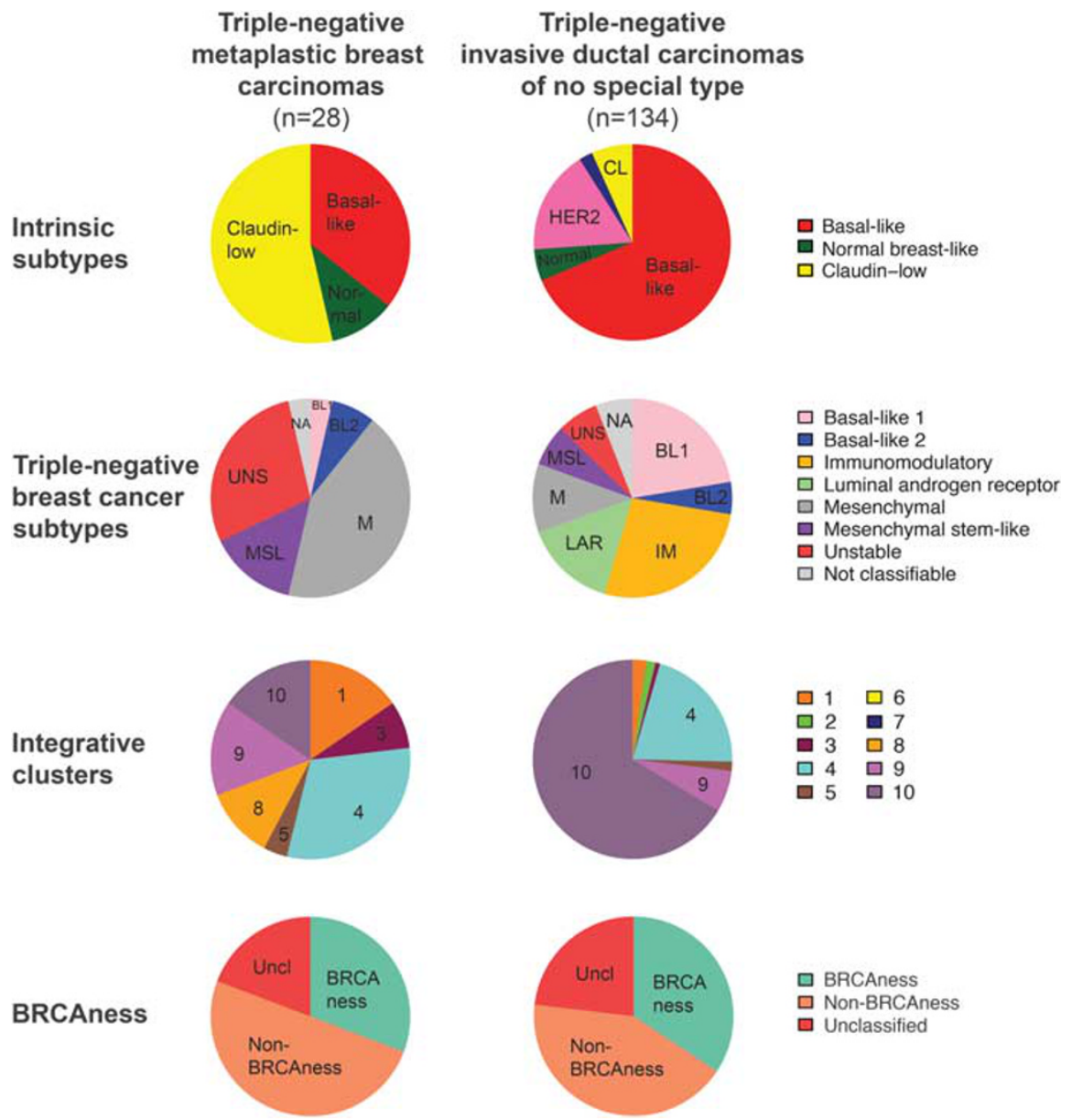

Figure 4 Triple-negative metaplastic breast cancers and invasive ductal carcinomas of no special type display distinct molecular classification. Pie charts represent the intrinsic molecular subtypes, triple-negative breast cancer subtypes, the integrative clusters and the BRCAness composition of triple-negative metaplastic breast cancers and of triple-negative invasive ductal carcinomas of no special type. For integrative cluster and BRCAness analysis, only metaplastic breast carcinomas with available SNP data were included $(n=26)$.

subset $(15 \%)$ of metaplastic breast carcinomas were of this integrative cluster (Fisher's exact test, $P<0.001)$. Furthermore, the low histologic gradeassociated IntClust 8 was not identified in triplenegative invasive ductal carcinomas of no special type (0/134), whereas $12 \%$ of metaplastic breast carcinomas were of this integrative cluster type (Fisher's exact test, $P=0.0039$; Figure 4). The frequency of cases displaying a BRCAness genomic signature was similar in metaplastic breast carcinomas $(31 \%)$ and in triple-negative invasive ductal carcinomas of no special type (34\%, Figure 4).

These data demonstrate that the distributions of molecular subtypes of triple-negative metaplastic breast carcinomas are distinct from those of triplenegative invasive ductal carcinomas of no special type. In addition, our findings provide evidence to suggest that metaplastic breast carcinomas are more homogeneous at the molecular level than triplenegative invasive ductal carcinomas of no special type, as the number of molecular subtypes identified in metaplastic breast carcinomas seems to be more limited than that of invasive ductal carcinomas of no special type.

\section{Discussion}

Metaplastic carcinoma of the breast is a morphologically heterogeneous group of tumors. ${ }^{13}$ Here we demonstrate that metaplastic breast carcinomas are also heterogeneous at the molecular level, and that the histologic diversity of metaplastic breast carcinomas impacts on the results of current microarraybased classifiers. In support of work performed previously by our group and others, ${ }^{18-20}$ we found metaplastic breast carcinomas to be either of claudin-low, basal-like, or normal breast-like subtypes. In addition, in the current study, we observed that metaplastic breast carcinomas were preferentially of mesenchymal-like and mesenchymal stemlike triple-negative breast cancer subtypes, using the triple-negative breast cancer molecular classification, of IntClust 4, IntClust 1, IntClust 8, and 
IntClust 9 based on the integrative clustering approach, and that these cancers not commonly display a BRCAness signature.

Our data provide evidence to suggest that the different histologic components of metaplastic breast carcinomas are associated with specific molecular features. When focusing on the metaplastic component present in a given frozen sample and subjected to molecular profiling, rather than on the overall diagnosis of a case, we observed that samples exclusively or predominantly composed of areas of spindle cell metaplasia or chondroid metaplasia were of claudin-low intrinsic molecular subtype and of mesenchymal-like triple-negative breast cancer subtype, respectively, whereas those samples exclusively or predominantly composed of squamous metaplasia were more heterogeneous. These findings imply that the molecular subtype of a metaplastic breast carcinoma may be different depending on the morphologic component sampled and subjected to molecular analysis. This finding expands on our previous observations that in a subset of metaplastic breast carcinomas, the different components of a case, despite being clonal and harboring identical TP53 mutations, displayed distinct copy number aberrations including focal high-level amplifications. ${ }^{24}$ It is currently unknown, however, whether the different histologic components of metaplastic breast carcinomas are underpinned by distinct constellations of point mutations, structural genetic rearrangements, and/ or epigenetic alterations. In fact, Hennessy et $a l^{20}$ observed that metaplastic breast carcinomas 'with squamous and sarcomatoid metaplasia' had a higher prevalence of PIK3CA mutations than other triple-negative breast carcinomas (47.4 vs $8.3 \%)$. These results are consistent with our observations, given that metaplastic breast carcinomas with sarcomatoid elements often display a mesenchymal-like or mesenchymal stem-like triple-negative breast cancer subtype and that PIK3CA mutations have been reported in $25 \%$ of cell line models of triple-negative breast cancers of mesenchymal-like and mesenchymal stem-like subtypes. ${ }^{3}$

Using xenograft models established from breast cancer cell lines representative of the different triple-negative breast cancer subtypes, Lehmann et $a l^{3}$ reported their differential sensitivity to chemotherapeutic, endocrine, and targeted agents. For example, xenografts of breast cancer cell lines classified as of mesenchymal-like subtype showed increased responses to the dual PI3K/mTOR inhibitor NVP-BEZ235, whereas xenografts of breast cancer cell lines classified as of basal-like subtype were sensitive to chemotherapy treatment. ${ }^{3}$ The triple-negative breast cancer subtypes were also reported to be predictive of pathologic complete response in breast cancer patients treated with neoadjuvant chemotherapy. ${ }^{36}$ In particular, patients with basal-like 1 breast cancers had a higher pathologic complete response rate $(52 \%)$ than patients with triple-negative breast carcinomas classified as of other subtypes. ${ }^{36}$ Consistent with these findings, Prat et $a l^{2}$ reported that tumors of claudin-low intrinsic subtype had a lower pathologic complete response rate $(38.9 \%)$ after neoadjuvant chemotherapy than basal-like cancers. Here, only one metaplastic breast carcinoma was classified as of basal-like 1 triple-negative breast cancer subtype, all metaplastic breast carcinomas with chondroid metaplasia were of mesenchymal-like triple-negative breast cancer subtype, and all metaplastic breast carcinomas with spindle cell metaplasia were of claudin-low intrinsic molecular subtype. Our results provide a potential molecular basis for the observation ${ }^{14}$ that metaplastic breast carcinomas less frequently respond to conventional chemotherapy regimens than other types of triplenegative breast carcinomas. Furthermore, it is plausible that different treatment strategies may be effective in different metaplastic breast carcinoma subtypes; however, further studies are required to assess the association of treatment response with the type of metaplastic breast carcinoma.

Triple-negative invasive ductal carcinomas of no special type have been shown to be heterogeneous at the molecular level and all triple-negative breast cancer subtypes can be identified in these tumors. ${ }^{3,10}$ Our data not only confirm these findings but also show that triple-negative metaplastic breast carcinomas are classified differently from triplenegative invasive ductal carcinomas of no special type. In particular, whereas the majority of triplenegative invasive ductal carcinomas of no special type were classified as of basal-like subtype, the majority of metaplastic breast carcinomas were classified as of claudin-low subtype. The luminal androgen receptor and immunomodulatory subtypes, consistently identified in cohorts of triplenegative invasive ductal carcinomas of no special type, were not found in the metaplastic breast carcinomas analyzed in this study. The distribution of IntClust subtypes was also found to be distinct. Unlike triple-negative invasive ductal carcinomas of no special type that are most frequently classified as of IntClust 10, as a group metaplastic breast carcinomas were found to be most frequently classified as of IntClust 4; however, the latter was shown to be dependent upon the metaplastic breast carcinoma subtype. A third of the metaplastic breast carcinomas studied here harbored chromosomal alterations (ie, tumor ploidy and number of large-scale state transitions) associated with BRCA1/2 inactivation (ie BRCAness signature), ${ }^{4}$ and a similar percentage of triple-negative invasive ductal carcinomas of no special type $(34 \%)$ also displayed this BRCAness signature.

Our results support earlier findings that the basal-like subtype, as defined by the intrinsic molecular classification (ie, using a research version of PAM50), and the triple-negative breast cancer basal-like 1 and basal-like 2 subtypes, as defined by 
the triple-negative breast cancer breast cancer classification, are not identical. ${ }^{3,10}$ We also observed that only a subset of the intrinsic claudin-low breast cancers, a subtype reported to be characterized by the enrichment of epithelial-to-mesenchymal transition markers and cancer stem cell-like features, ${ }^{2}$ are of triple-negative breast cancer mesenchymal-like $(19 \%)$ or mesenchymal stem-like $(25 \%)$ subtypes.

This study has several limitations. For molecular analysis, we only had one histologic component per metaplastic breast carcinoma available, and we were unable to assess whether different histologic components of a given case would have distinct molecular subtypes. In addition, we were unable to test whether the histologic subtype of metaplastic breast carcinoma would have an impact on the survival of metaplastic breast carcinoma patients, as survival data were not available for the vast majority of cases included in this study. Further analyses to ascertain whether the outcome of patients with metaplastic breast carcinoma would vary according to their molecular subtype are warranted.

Taken together, here we demonstrate that the diversity of histologic features of metaplastic breast carcinomas is reflected at the molecular level. At present, the information provided by histologic classification and grading of metaplastic breast carcinomas is arguably more clinically relevant than that provided by molecular profiling of these tumors without any histologic stratification, given that entities defined on the basis of histology (eg, lowgrade fibromatosis-like metaplastic carcinoma ${ }^{32,33}$ ) display a clinical behavior that differs from that of the remaining types of metaplastic breast carcinomas. In this era of precision medicine, our study also highlights the importance of careful histologic analysis of breast cancers, given that histologic subtyping of metaplastic breast carcinomas is likely of importance in studies aiming to identify the drivers of and molecular targets for this disease, as subgroups of metaplastic breast carcinomas with different histologic components may have distinct molecular features, biological characteristics, and responses to therapy.

\section{Acknowledgments}

$\mathrm{RN}$ is funded by a Breast Cancer Campaign Career Development Fellowship; RS is funded by the Biostatistics core grant (P30 CA008748).

\section{Disclosure/conflict of interest}

Tatiana Popova and Marc-Henri Stern are named inventors on a patent filed by Institut Curie based on the method for the identification of BRCAness by genomic profiling, with exclusive licensing to Myriad Genetics. The remaining authors declare no conflict of interest.

\section{References}

1 Parker JS, Mullins M, Cheang MC, et al. Supervised risk predictor of breast cancer based on intrinsic subtypes. J Clin Oncol 2009;27:1160-1167.

2 Prat A, Parker JS, Karginova O, et al. Phenotypic and molecular characterization of the claudin-low intrinsic subtype of breast cancer. Breast Cancer Res 2010; 12:R68.

3 Lehmann BD, Bauer JA, Chen X, et al. Identification of human triple-negative breast cancer subtypes and preclinical models for selection of targeted therapies. J Clin Invest 2011;121:2750-2767.

4 Popova T, Manie E, Rieunier G, et al. Ploidy and largescale genomic instability consistently identify basallike breast carcinomas with BRCA1/2 inactivation. Cancer Res 2012;72:5454-5462.

5 Curtis C, Shah SP, Chin SF, et al. The genomic and transcriptomic architecture of 2,000 breast tumours reveals novel subgroups. Nature 2012;486:346-352.

6 Metzger-Filho O, Tutt A, de Azambuja E, et al. Dissecting the heterogeneity of triple-negative breast cancer. J Clin Oncol 2012;30:1879-1887.

7 Mayer IA, Abramson VG, Lehmann BD, et al. New strategies for triple-negative breast cancer-deciphering the heterogeneity. Clin Cancer Res 2014;20:782-790.

8 Foulkes WD, Smith IE, Reis-Filho JS. Triple-negative breast cancer. N Engl J Med 2010;363:1938-1948.

9 Turner NC, Reis-Filho JS. Tackling the diversity of triple-negative breast cancer. Clin Cancer Res 2013;19: 6380-6388.

10 Lehmann BD, Pietenpol JA. Identification and use of biomarkers in treatment strategies for triple-negative breast cancer subtypes. J Pathol 2014;232:142-150.

11 Weigelt B, Geyer FC, Reis-Filho JS. Histological types of breast cancer: how special are they? Mol Oncol 2010;4:192-208.

12 Lakhani SR, Ellis IO, Schnitt SJ, et al. Special subtypes, In: WHO Classification of Tumours of the Breast, 4th edn. IARC Press: Lyon, 2012, pp 39-76.

13 Weigelt B, Eberle C, Cowell CF, et al. Metaplastic breast carcinoma: more than a special type. Nat Rev Cancer 2014;14:147-148.

14 Hennessy BT, Giordano S, Broglio K, et al. Biphasic metaplastic sarcomatoid carcinoma of the breast. Ann Oncol 2006;17:605-613.

15 McCarthy A, Savage K, Gabriel A, et al. A mouse model of basal-like breast carcinoma with metaplastic elements. J Pathol 2007;211:389-398.

16 Molyneux G, Geyer FC, Magnay FA, et al. BRCA1 basal-like breast cancers originate from luminal epithelial progenitors and not from basal stem cells. Cell Stem Cell 2010;7:403-417.

17 Liu X, Holstege H, van der Gulden H, et al. Somatic loss of BRCA1 and p53 in mice induces mammary tumors with features of human BRCA1-mutated basallike breast cancer. Proc Natl Acad Sci USA 2007;104: 12111-12116.

18 Weigelt B, Horlings HM, Kreike B, et al. Refinement of breast cancer classification by molecular characterization of histological special types. J Pathol 2008;216: 141-150.

19 Weigelt B, Kreike B, Reis-Filho JS. Metaplastic breast carcinomas are basal-like breast cancers: a genomic profiling analysis. Breast Cancer Res Treat 2009;117:273-280.

20 Hennessy BT, Gonzalez-Angulo AM, Stemke-Hale K, et al. Characterization of a naturally occurring breast 
cancer subset enriched in epithelial-to-mesenchymal transition and stem cell characteristics. Cancer Res 2009;69:4116-4124.

21 Elston CW, Ellis IO. Pathological prognostic factors in breast cancer. I. The value of histological grade in breast cancer: experience from a large study with longterm follow-up. Histopathology 1991;19:403-410.

22 Marchio C, Iravani M, Natrajan R, et al. Genomic and immunophenotypical characterization of pure micropapillary carcinomas of the breast. J Pathol 2008;215:398-410.

23 Tan DS, Lambros MB, Rayter S, et al. PPM1D is a potential therapeutic target in ovarian clear cell carcinomas. Clin Cancer Res 2009;15:2269-2280.

24 Geyer FC, Weigelt B, Natrajan R, et al. Molecular analysis reveals a genetic basis for the phenotypic diversity of metaplastic breast carcinomas. J Pathol 2010;220:562-573.

25 Carter SL, Cibulskis K, Helman E, et al. Absolute quantification of somatic DNA alterations in human cancer. Nat Biotechnol 2012;30:413-421.

26 Hammond ME, Hayes DF, Dowsett M, et al. American Society of Clinical Oncology/College Of American Pathologists guideline recommendations for immunohistochemical testing of estrogen and progesterone receptors in breast cancer. J Clin Oncol 2010;28: 2784-2795.

27 Wolff AC, Hammond ME, Hicks DG, et al. Recommendations for human epidermal growth factor receptor 2 testing in breast cancer: American Society of Clinical Oncology/College of American Pathologists clinical practice guideline update. J Clin Oncol 2013;31:3997-4013.
28 Grigoriadis A, Mackay A, Noel E, et al. Molecular characterisation of cell line models for triple-negative breast cancers. BMC Genomics 2012;13:619.

29 Barbosa-Morais NL, Dunning MJ, Samarajiwa SA, et al. A re-annotation pipeline for Illumina BeadArrays: improving the interpretation of gene expression data. Nucleic Acids Res 2010;38:e17.

30 Popova T, Manie E, Stoppa-Lyonnet D, et al. Genome Alteration Print (GAP): a tool to visualize and mine complex cancer genomic profiles obtained by SNP arrays. Genome Biol 2009;10:R128.

31 Tse GM, Tan PH, Putti TC, et al. Metaplastic carcinoma of the breast: a clinicopathological review. J Clin Pathol 2006;59:1079-1083.

32 Sneige N, Yaziji H, Mandavilli SR, et al. Low-grade (fibromatosis-like) spindle cell carcinoma of the breast. Am J Surg Pathol 2001;25:1009-1016.

33 Gobbi H, Simpson JF, Borowsky A, et al. Metaplastic breast tumors with a dominant fibromatosis-like phenotype have a high risk of local recurrence. Cancer 1999;85:2170-2182.

34 Dawson SJ, Rueda OM, Aparicio S, et al. A new genome-driven integrated classification of breast cancer and its implications. EMBO J 2013;32:617-628.

35 Elloumi F, Hu Z, Li Y, et al. Systematic bias in genomic classification due to contaminating non-neoplastic tissue in breast tumor samples. BMC Med Genomics 2011;4:54.

36 Masuda H, Baggerly KA, Wang Y, et al. Differential response to neoadjuvant chemotherapy among 7 triplenegative breast cancer molecular subtypes. Clin Cancer Res 2013;19:5533-5540.

Supplementary Information accompanies the paper on Modern Pathology website (http://www.nature.com/ modpathol) 